\title{
Mathematical formulation to predict the harmonics of the superconducting Large Hadron Collider magnets. II. Dynamic field changes and scaling laws
}

\author{
Nicholas J. Sammut \\ CERN-The European Organisation for Nuclear Research, CH-1211 Geneva 23, Switzerland, \\ and UOM-The University of Malta, Msida MSD 2080, Malta \\ Luca Bottura \\ CERN-The European Organisation for Nuclear Research, CH-1211 Geneva 23, Switzerland \\ Pierre Bauer \\ EFDA-CSU, The European Fusion Development Agreement Close Support Unit, 85748 Garching, Germany \\ George Velev \\ Fermilab-Fermi National Accelerator Laboratory, Batavia, Illinois, USA 60510-5011 \\ Tatiana Pieloni \\ CERN-The European Organisation for Nuclear Research, CH-1211 Geneva 23, Switzerland \\ Joseph Micallef \\ UOM-The University of Malta, Msida MSD 2080, Malta
}

(Received 7 May 2007; published 31 August 2007)

\begin{abstract}
A superconducting particle accelerator like the LHC (Large Hadron Collider) at CERN, can only be controlled well if the effects of the magnetic field multipoles on the beam are compensated. The demands on a control system solely based on beam feedback may be too high for the requirements to be reached at the specified bandwidth and accuracy. Therefore, we designed a suitable field description for the LHC (FIDEL) as part of the machine control baseline to act as a feed-forward magnetic field prediction system. FIDEL consists of a physical and empirical parametric field model based on magnetic measurements at warm and in cryogenic conditions. The performance of FIDEL is particularly critical at injection when the field decays, and in the initial part of the acceleration when the field snaps back. These dynamic components are both current and time dependent and are not reproducible from cycle to cycle since they also depend on the magnet powering history. In this paper a qualitative and quantitative description of the dynamic field behavior substantiated by a set of scaling laws is presented.
\end{abstract}

DOI: 10.1103/PhysRevSTAB.10.082802

PACS numbers: 85.70.Ay, 41.85.Lc, 07.55.Db

\section{INTRODUCTION}

The baseline of the LHC control system includes feedforward control intended to reduce the burden on the beam based feedback. Known as the field description for the LHC (FIDEL) [1], this feed-forward system will predict the main field and the harmonics of the superconducting magnets during the whole machine operation cycle. This system is particularly critical during the beam injection and the initial phase of the particle acceleration where the machine magnetic state is dynamic and its reproducibility is, to some extent, unknown.

During beam injection, the LHC superconducting magnets need to have a constant magnetic field of $0.537 \mathrm{~T}$ and therefore are kept at a constant current of $760 \mathrm{~A}$. However, the magnetic field multipoles drift when the magnets are on a constant current plateau. This appears as a "decay" of the persistent current contribution to the multipoles and causes significant changes in the beam tune and machine chromaticity [2]. The present understanding of the origin of this dynamic magnetic behavior is the diffusion of a nonuniform current distribution along the Rutherford cable originating from spatial gradients in the field sweep rate and gradients in the cable properties (e.g. cross-contact resistances). Even at constant transport current, as is the case on the injection plateau, these currents produce spatially modulated changes in the local field. These field changes locally reduce the magnetization and hence cause the decay [3-7].

In turn, when the external field is increased during the first few seconds of the current ramp, the magnetization is restored to its original hysteresis state, hence canceling out the decay. This phase, called snap-back [8], can be too fast to be compensated solely using beam diagnostics.

In addition, these dynamic field changes are not reproducible from one powering cycle to another and they are dependent on the powering history of the magnet [9].

Extensive research on these phenomena has been done at the hadron electron ring facility (HERA) $[10,11]$ and for the superconducting super collider (SSC) [12]. These ef- 
fects were also measured at the relativistic heavy ion collider (RHIC) [13]. The aim of this paper is not to investigate the physical origin of these effects but to present a general model of them based on cryogenic magnetic measurements. In addition, through the use of simple but effective scaling laws, it will be shown that this dynamic model is universal and can be applied to one single magnet as well as extrapolated to the whole magnet population. The scope of the study is limited to the dipole magnets of which a large sample has been measured and substantial statistics have been performed. The attention is restricted to the first allowed multipoles $b_{1}, b_{3}$, and $b_{5}$, for which the effects are systematic. Where relevant, results from other magnet productions are quoted, and, in particular, the experience at Fermilab on the Tevatron dipoles, to which CERN has participated actively, and from the HERA reference magnets that have been extensively exploited to steer and optimize operation.

\section{TIME DEPENDENCE OF DECAY}

\section{A. Time dependence in LHC dipoles}

The LHC dipole magnets have a cost-saving twinaperture design, where two particle beam apertures with separate coil systems are incorporated within the same magnet [14]. The standard decay magnetic measurements executed on dipoles consist of rotating coil measurements [15] in both apertures during a $1000 \mathrm{~s}$ simulated particle injection plateau at $0.537 \mathrm{~T}$. The injection conditions are reached following a standard powering cycle consisting of a cleansing quench, a ramp to $8.33 \mathrm{~T}$ at $50 \mathrm{~A} / \mathrm{s}$, a $1000 \mathrm{~s}$ flattop, and a ramp-down to $0.25 \mathrm{~T}$ at $50 \mathrm{~A} / \mathrm{s}$. The purpose of this precycle is to simulate the LHC operation at $7 \mathrm{TeV}$, while the purpose of the cleansing quench is to erase the memory of previous powering cycles and thus make the measurements comparable. The sample measured consists of 352 apertures (corresponding to 176 magnets) and is almost equally distributed amongst the three different manufacturers (Alstom ${ }^{\circledR}$, Ansaldo Superconduttori ${ }^{\circledR}$, and Babcock Noell@).

As generally accepted for accelerator magnets and for use in beam optics simulations, the magnetic field $\boldsymbol{B}$ in the 2D imaginary plane $(x, y)$ can be expressed using the harmonic expansion:

$$
\boldsymbol{B}(x, y)=\sum_{n=1}^{\infty} \boldsymbol{C}_{n}\left(\frac{x+i y}{R_{\mathrm{ref}}}\right)^{n-1},
$$

where $\boldsymbol{C}_{n}$ indicates the generic non-normalized complex harmonic of order $n$ given in the reference frame aligned with the main field direction. $R_{\text {ref }}$ is the reference radius $(=17 \mathrm{~mm}$ for the LHC) and is representative of the maximum beam size. For convenience, the normalized harmonic coefficients, indicated as $c_{n}$, can be defined as

$$
\boldsymbol{c}_{n}=b_{n}+i a_{n}=10^{4} \frac{\boldsymbol{C}_{n}}{\boldsymbol{B}_{m}} .
$$

$\boldsymbol{B}_{m}$ is the main magnetic field expressed in a reference frame where the main skew component is zero. $b_{n}$ and $a_{n}$ are the normal and skewed multipole coefficients, respectively. The factor $10^{4}$ is used to produce practical relative dimensions for the normalized coefficients. The normalized $\boldsymbol{c}_{n}$ are expressed in the form above in so called "units."

Figure 1 shows the variation of $b_{1}, b_{3}$, and $b_{5}$ during injection, arbitrarily shifted along the vertical axis to make the initial value at injection equal to zero. Note that only 58 apertures are included in Fig. 1 so as to limit the amount of data in one graph. However, the average decay is computed from the entire magnet population.

A quantity of specific interest to analyze the properties of the magnet population is the decay amplitude at the end of the injection. This is summarized in Fig. 2 and Table I, reporting, respectively, the average decay amplitude $\delta_{\text {std }}$ of the main field and the harmonics. It should be noted that in Table I the entry "(unit)" means the ratio of the multipole
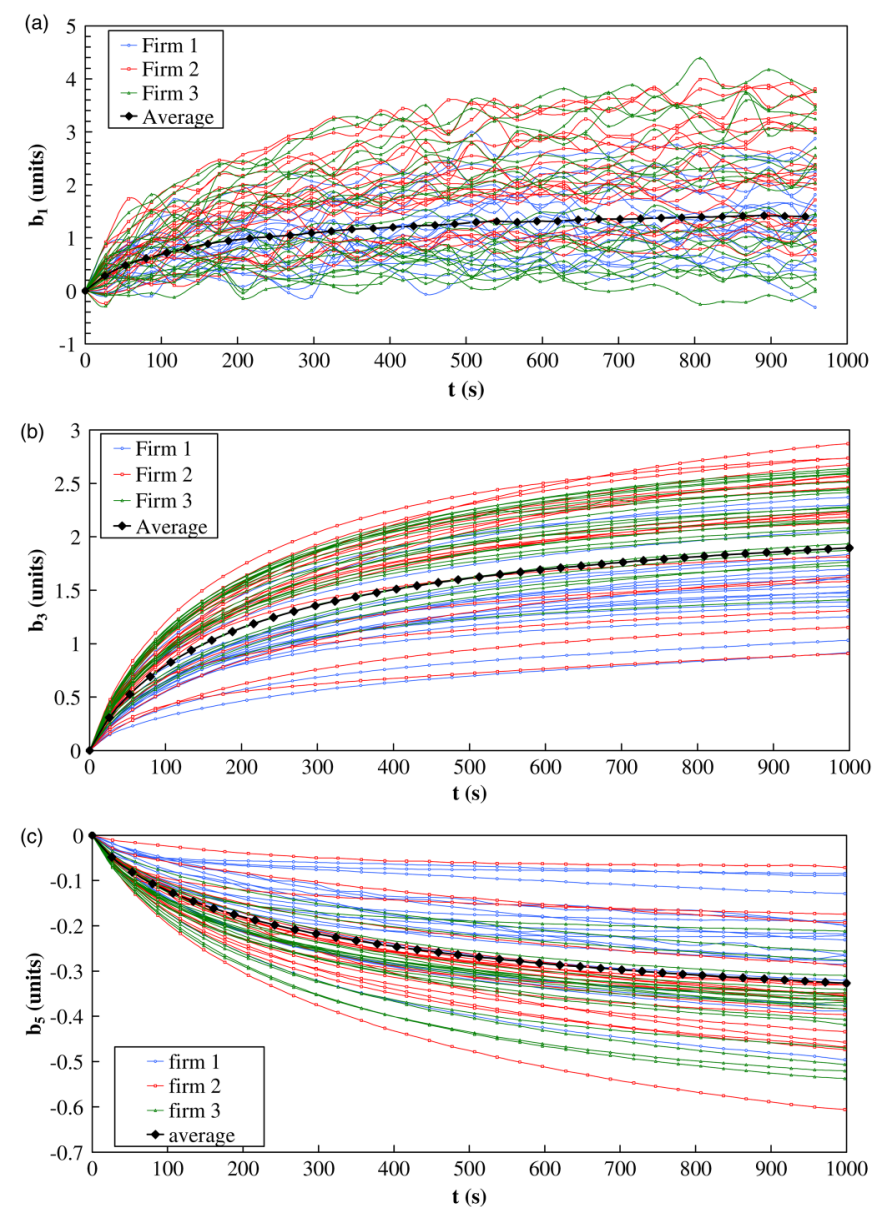

FIG. 1. (Color) (a) Decay of $b_{1}$, (b) $b_{3}$, and (c) $b_{5}$ (units @ $17 \mathrm{~mm}$ ), measured during a $1000 \mathrm{~s}$ simulated injection plateau following a standard cleansing cycle. The values have been shifted arbitrarily along the $y$-axis to cancel the initial value that is magnet dependent. 


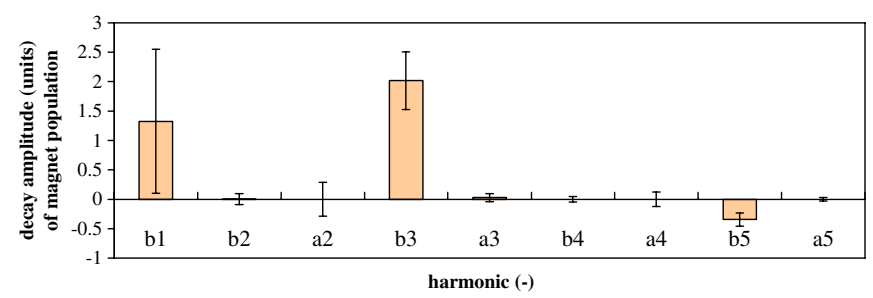

FIG. 2. (Color) The average decay amplitude of the main field and the harmonics after $1000 \mathrm{~s}$.

TABLE I. The average decay amplitude at $1000 \mathrm{~s}$ for the 352 apertures and the $99 \%$ confidence interval for the allowed harmonics.

\begin{tabular}{lcccc}
\hline \hline & Dimension & $b_{1}$ & $b_{3}$ & $b_{5}$ \\
\hline $\begin{array}{c}\text { Average decay amplitude } \\
\text { after } 1000 \mathrm{~s}\left(\delta_{\text {std }}\right)\end{array}$ & (units) & 1.41 & 2.01 & -0.34 \\
\begin{tabular}{c}
$99 \%$ confidence interval \\
\hline \hline
\end{tabular} & (units) & 0.217 & 0.07 & 0.02 \\
\hline
\end{tabular}

to the dipole field at $17 \mathrm{~mm}$ reference radius multiplied by $10^{-4}$ as indicated in Eq. (2).

From Fig. 2 we clearly see that the decay manifests itself as a systematic behavior only in the allowed harmonics, and hence it must be modeled and compensated in the machine. From this observation, the decay modeling is limited to the main field and the first two allowed harmonics which can be compensated by using corrector magnets.

We use our understanding of the physical origin of the decay to develop a mathematical formulation that can be derived to describe the decay evolution in time. In particular, we assume that the decay driver is current diffusion in the superconducting cable. Making the hypothesis that the cable current distributes continuously among the strands of a uniform cable, the time evolution of the currents is governed by an infinite series of harmonic modes damped by an exponential with time constants $\tau_{n}=\frac{\tau}{(2 n-1)^{2}}$ [3]. The time constants depend on the cable geometry (affecting the line inductance) and the interstrand resistances. A direct solution of current diffusion is not practical, as it depends on too many parameters that are not measured (such as the cross-contact resistance and its variation along the coils). In our model we substitute these unknowns by constants that can be determined by fitting to cold magnetic measurement data. Under these assumptions, the normalized decay can be modeled by the following equation:

$$
\begin{aligned}
\Delta\left(t, t_{\mathrm{inj}}, \tau, d\right)= & d\left(1-e^{\left(t_{\mathrm{inj}}-t\right) / \tau}\right) \\
& +(1-d)\left(1-e^{\left(t_{\mathrm{inj}}-t\right) /(9 \tau)}\right)+\text { HOT, }
\end{aligned}
$$

which holds for $I=I_{\mathrm{inj}}$ and $t>t_{\mathrm{inj}} . t$ is the instantaneous time, $t_{\text {inj }}$ is the time when injection starts, $I_{\text {inj }}$ is the current at injection, $\tau$ is the time constant, and HOT stands for higher order terms in the series expansion. Neglecting this last, the parameter $d$ gives the normalized weight of the fast mode of the decay and its complement to one, $1-d$, gives the normalized weight of the slow mode. In practice, using Eq. (3) the main field decay is given by

$$
B_{m}^{\text {decay }}=\delta_{m} \frac{B_{b_{\mathrm{inj}}}}{10^{4}} \frac{I_{\mathrm{inj}}}{|I|} \frac{\Delta\left(t, t_{\mathrm{inj}}, \tau_{m}, d_{m}\right)}{\Delta\left(t_{\mathrm{inj}}^{\mathrm{sdd}}, t_{\mathrm{inj}}, \tau_{m}, d_{m}\right)},
$$

where the parameter $\delta_{m}$ represents the decay amplitude at a reference time $t_{\mathrm{inj}}^{\mathrm{std}} . B_{b_{\mathrm{inj}}}$ is the field at the beginning of injection, $I_{\text {inj }}$ is the injection current. The contribution of decay to the transfer function is modeled by

$$
\mathrm{TF}^{\text {decay }}=\delta_{m} \frac{B_{b_{\text {inj }}}}{10^{4}} \frac{I_{\text {inj }}}{|I|^{2}} \frac{\Delta\left(t, t_{\text {inj }}, \tau_{m}, d_{m}\right)}{\Delta\left(t_{\mathrm{inj}}^{\mathrm{std}}, t_{\mathrm{inj}}, \tau_{m}, d_{m}\right)},
$$

where the transfer function (TF) is defined as the ratio of field generated and operating current:

$$
\mathrm{TF}=\frac{B_{m}}{I}
$$

The contribution to the harmonics is given by

$$
c_{n}^{\text {decay }}=\delta_{n} \frac{I_{\mathrm{inj}}}{|I|} \frac{\Delta\left(t, t_{\mathrm{inj}}, \tau_{n}, d_{n}\right)}{\Delta\left(t_{\mathrm{inj}}^{\mathrm{sdd}}, t_{\mathrm{inj}}, \tau_{n}, d_{n}\right)}
$$

where $\delta_{m}$ and $\delta_{n}$ are in units.

Figure 3 shows the decay model for $b_{3}$ for an injection plateau of $10000 \mathrm{~s}$. The values of the parameters obtained as a result of the fits of the average decay as well as the standard deviation of the difference between the sample average and the model are reported in Table II. The two-

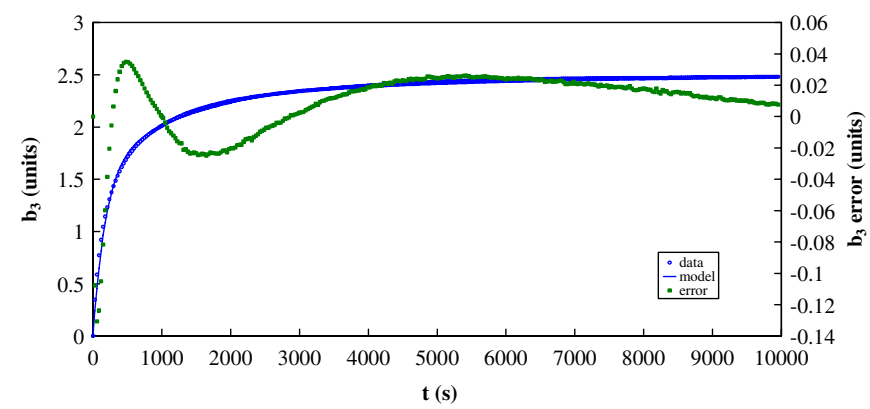

FIG. 3. (Color) The $b_{3}$ decay model of one aperture on an injection plateau of $10000 \mathrm{~s}$. The residual error between the model and the data is below 0.1 units over the whole range.

TABLE II. Parameters obtained fitting the model of Eqs. (3)(7) to the average decay in the population analyzed, representing the behavior of the LHC.

\begin{tabular}{lcccc}
\hline \hline Parameter & Dimension & $b_{1}$ & $b_{3}$ & $b_{5}$ \\
\hline$\tau$ & $(\mathrm{s})$ & 227.58 & 189.04 & 284.15 \\
$d$ & $(\cdots)$ & 0.978 & 0.660 & 0.660 \\
$\delta$ & (units) & 1.41 & 2.01 & -0.34 \\
\hline Max residual error & (units) & 0.32 & 0.13 & 0.04 \\
\hline \hline
\end{tabular}


terms approximation described above is enough to maintain the residual fit error to acceptable bounds, i.e., 0.32 units for $b_{1}, 0.13$ units for $b_{3}$, and 0.04 units for $b_{5}$.

\section{B. Decay scaling}

So far we have discussed modeling of a finite population in one specific cycling condition. Operation in the LHC will depend on many factors that will surely cause deviations from the measurement conditions used during cold tests. For this reason, it is planned to improve the model using data from direct beam measurements as well as offline reference magnet measurements. The adjustment will be effective only if the model of the average or a reference magnet measurement can be scaled to be representative of the whole magnet population, which is not obvious in principle. Observing the single magnet data, it seems that a simple scaling factor applied to the decay of a single magnet, i.e. stretching the measured data in the $y$ direction, could be enough to match the average curve. This is clearly true if the dynamics of the decay do not change from magnet to magnet. Starting with this assumption, it was sought whether the scaling law,

$$
\delta_{n}=f_{n}^{\text {decay }} \cdot \delta_{n}^{i}
$$

produces a satisfactory result. In Eq. (8) $\delta_{n}$ is the average decay (i.e. the value for the sector or for the ring), $\delta_{n}^{i}$ is the decay of the reference magnet $i$, and $f_{n}^{\text {decay }}$ is the scaling factor. The latter is determined as the ratio of the measured decays for the sample average and for the reference magnet chosen at the end of the simulated injection, i.e., in the above notation:

$$
f_{n}^{\text {decay }}=\frac{\delta_{n}}{\delta_{n}^{i}} \quad \text { at } t=1000 \mathrm{~s} .
$$

It should be noted that there is no free parameter in the above scaling, all quantities being known once the measurement on the beam is performed or once the reference magnet, or a suitable sample, have been measured in cold conditions.

Equations (8) and (9) have been used to scale the decay of each magnet measured, producing curves of the type

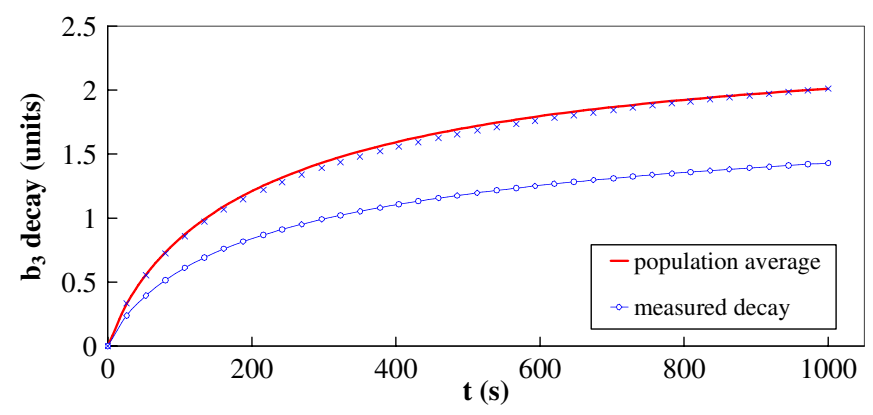

FIG. 4. (Color) Example of scaling and comparison of scaled sextupole decays in magnet 3154, aperture 1 . represented in Fig. 4 for a selected magnet (in this case the sextupole harmonic of magnet 3154 aperture 1). The difference between the scaled decay and the average of the magnet population has been computed at all times during the injection plateau. To quantify the goodness of the scaling, we have taken the maximum of the absolute value of this difference.

A histogram of the maximum residual error of all the magnets as well as a log-normal distribution for $b_{1}, b_{3}$, and $b_{5}$, respectively, are shown in Fig. 5. The log-normal distribution is used because it can fit a data set that is skewed and can also be used to describe data that cannot fall below zero but that might increase without limit. The goodness of fit is tested using the Kolmogorov-Smirnov test [16], which is satisfied for $b_{3}$ and $b_{5} . b_{1}$ does not pass the test, and we attribute this to the noise inherent in the measurement (see Fig. 1).

The scaling law tested produces typical maximum residual scaling errors in the range 0.1 to 5 units @ $17 \mathrm{~mm}$ for
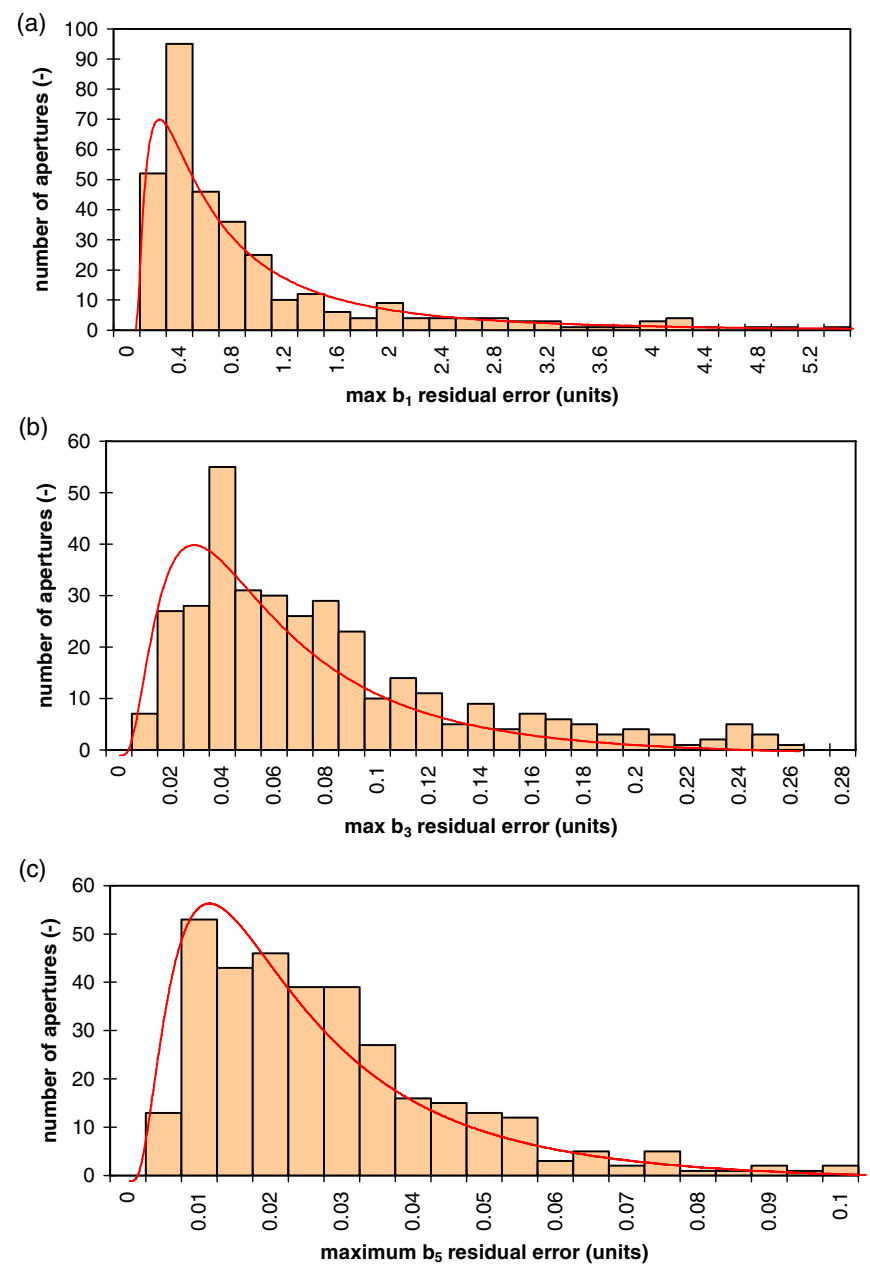

FIG. 5. (Color) (a), (b), (c) Histograms and log-normal distribution of the maximum residual error for $b_{1}, b_{3}$, and $b_{5}$, respectively, between the scaled harmonic decay and the average harmonic decay of the magnet set analyzed. 
$b_{1}, 0.01$ to 0.26 units @ $17 \mathrm{~mm}$ for $b_{3}$, and 0.005 to 0.1 units @ $17 \mathrm{~mm}$ for $b_{5}$. There are few outliers that are not shown in the figure. These are generally related to magnets that have a large scaling factor or that have anomalous behavior and that appear as a tail in the distributions.

Since the data distribution is skewed as shown in the histograms, the most probable residual errors (i.e. the mode) are less than the medians of the distribution. A conservative choice can be made by taking the median as an indication for the typical scaling, i.e., 0.5 units @ $17 \mathrm{~mm}$ for $b_{1}, 0.06$ units @ $17 \mathrm{~mm}$ for $b_{3}$ and 0.02 units @ $17 \mathrm{~mm}$ for $b_{5}$. In fact, in principle, it would be possible to achieve better results by defining the scaling factor based on a general optimization over the time span available in measured data. This is not done here to keep the reasoning simple and because it has little influence on the final conclusions.

\section{Tevatron dipoles}

As a part of the overall optimization of the Tevatron run II, several dipole magnets were remeasured at the magnet test facility in Fermilab $[17,18]$ aiming at reducing beam losses associated to residual correction errors during injection and snap-back. Thanks to the copious results obtained in this measurement campaign, it was possible to compare the behavior of the sextupole during injection in specific magnets to the chromaticity measurements taken during the injection plateau in the accelerator [19]. The result of this test is shown in Fig. 6, which demonstrates that the good agreement between the average behavior of a magnet population and the scaled results from a single magnet is not accidental.

In the case reported in Fig. 6, the scaled magnet behavior reproduces the dynamics of the Tevatron chromaticity evolution to within 0.04 units @ $25.4 \mathrm{~mm}$ over a time span of nearly 2 hours. Beyond this time, there is a deviation which is due to a difference in the dynamics of the

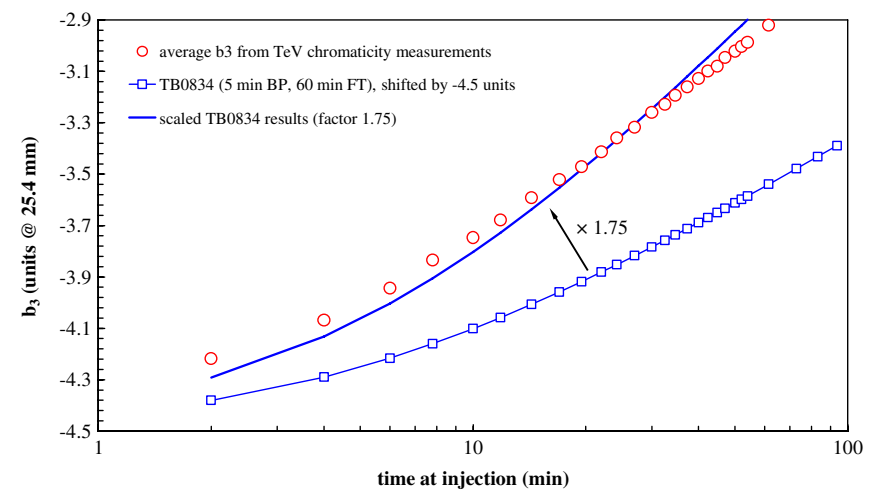

FIG. 6. (Color) Comparison of the sextupole deduced from chromaticity measurement during an injection at Tevatron, and the scaled measurements in a spare dipole. The scaling factor was optimized to minimize the residual error over the complete injection plateau, of 100 minutes. decays. However, the deviation remains small. This gives confidence that the scaling of Eq. (8) can produce results accurate enough for precise control.

\section{HERA dipoles}

The correction scheme employed by HERA at DESY makes use of online reference magnets and look-up tables. Two reference magnets, one for each magnet production line, have been chosen to represent the behavior of the two halves of the proton ring. The reference magnets were chosen to be at the center of the drift spread of their respective magnet family.

The beam parameters can be controlled automatically using NMR probes in the reference magnets to detect the $b_{1}$ change, and rotating coils to measure the drift of the $b_{3}$ component [20]. The corrections obtained are applied without scaling to the corrector magnets in the ring. This corresponds to the scaling procedure outlined above for the LHC magnets, where the scaling factor $f_{\text {decay }}$ of the single magnet to the average of the population is 1 because of the magnet selection adopted.

As shown in [21,22], the effect of decaying persistent currents leads to a change in the horizontal and vertical chromaticities in opposite directions. Without correction, the chromaticity reaches unacceptable values within a few minutes. However, if the correction system is switched on, the use of reference magnet data counteracts the decaying persistent current sextupole fields and the chromaticity in both planes is kept close to the desired values. As in the case of the Tevatron dipoles, these results show that a single magnet can be taken to represent the behavior of a whole family and support the scaling property observed for the LHC magnets.

\section{SNAP-BACK CORRELATION}

\section{A. LHC dipoles}

Figure 7 shows typical LHC snap-back curves for $b_{3}$, as measured on the dipole 2211. The vertical line at $760 \mathrm{~A}$ corresponds to the decay at constant current, while the snap-back is the change in $b_{3}$ that takes place during the first $50 \mathrm{~A}$ of the acceleration ramp, when the $b_{3}$ is observed

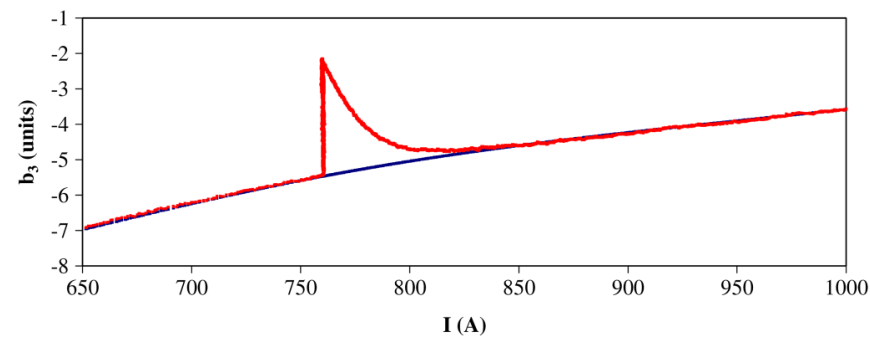

FIG. 7. (Color) (red) The $b_{3}$ decay during the injection plateau of an LHC cycle (at $760 \mathrm{~A}$ ) and the subsequent snap-back when the current begins to ramp, measured on LHC dipole 2211. (blue) $b_{3}$ hysteresis curve without an injection plateau. 
to return to the hysteresis branch that would have been measured without the injection stop (dashed line). The measurement of the snap-back was performed with a snap-back analyzer [23] that outperformed rotating coils [15] which are too slow to provide the time resolution necessary for accurate modelling ( 1 to $10 \mathrm{~Hz}$ ). A typical snap-back measurement campaign consists of several LHC cycles with the precycle parameters changed so as to vary the decay amplitude. The cycles are separated by a quench to erase the memory of previous powering.

It was found experimentally [24], and proven analytically in [25], that during the snap-back the first allowed harmonics $b_{3}$ and $b_{5}$ follow an exponential law. For the normal sextupole, this law was written as follows:

$$
b_{3}^{\text {snap-back }}(t)=b_{3}^{\text {decay }} e^{-\left[I(t)-I_{\text {inj }}\right] /(\Delta I)},
$$

where $b_{3}^{\text {snap-back }}(t)$ is the sextupole variation during the snap-back, $I(t)$ is the instantaneous value of the current, initially at the injection value $I_{\text {inj. }}$. The amplitude $b_{3}^{\text {decay }}$ of the snap-back and the current change $\Delta I$ are the two fitting constants. However, given that the multipoles are continuous in time, the snap-back amplitude is equal and opposite to the magnitude of the decay at the end of the injection. This implies that $b_{3}^{\text {decay }}$ is not an independent parameter in the overall model.

Figure 8 shows the exponential fit of the sextupole snapback data of Fig. 7, demonstrating that the model is well suited to the data. The standard deviation of the fit is in general less than 0.03 units during the whole snap-back. Based on this observation, the snap-back of the main field, transfer function, and all harmonics is modeled as follows:

$$
\begin{aligned}
B_{m}^{\text {snap-back }} & =B_{m}^{\text {decay }}\left(t_{\text {ramp }}\right) e^{\left(I_{\text {inj }}-I\right) /\left(\Delta I_{m}\right)} \\
\mathrm{TF}^{\text {snap-back }} & =\operatorname{TF}^{\text {decay }}\left(t_{\text {ramp }}\right) e^{\left(I_{\text {inj }}-I\right) /\left(\Delta I_{m}\right)} \\
c_{n}^{\text {snap-back }} & =c_{n}^{\text {decay }}\left(t_{\text {ramp }}\right) e^{\left(I_{\text {inj }}-I\right) /\left(\Delta I_{n}\right)},
\end{aligned}
$$

where the factors $B_{m}^{\text {decay }}\left(t_{\text {ramp }}\right), \quad \operatorname{TF}^{\text {decay }}\left(t_{\text {ramp }}\right)$, and $c_{n}^{\text {decay }}\left(t_{\text {ramp }}\right)$ are the change of the main field, the transfer function, and the normalized harmonics, respectively, during the decay evaluated at the time of the beginning of the

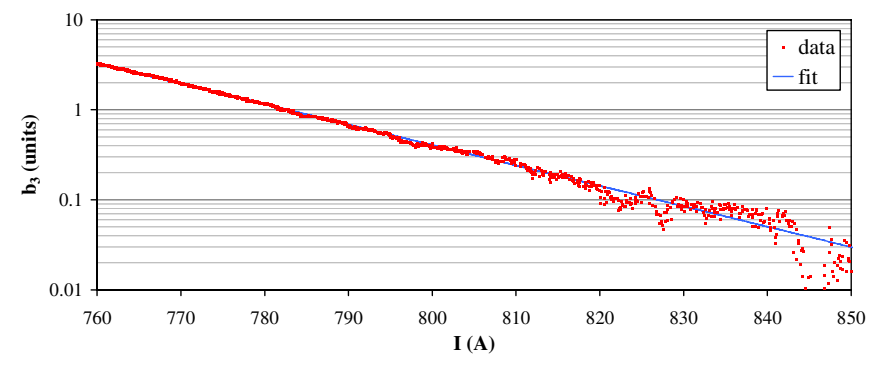

FIG. 8. (Color) Exponential fit of measured sextupole change during snap-back on the LHC dipole 2211. ramp $t_{\text {ramp. }}$. These parameters can hence be determined from the double exponential fit of Eq. (3). The only remaining parameter is the characteristic currents for the exponential change, $\Delta I_{m}$ and $\Delta I_{n}$.

Analyzing data obtained for a single magnet during measurements of snap-back following different magnet powering sequences, it can be observed that both the amplitude parameters $\left(B_{m}^{\text {decay }}\left(t_{\text {ramp }}\right), \operatorname{TF}^{\text {decay }}\left(t_{\text {ramp }}\right)\right.$, and $\left.c_{n}^{\text {decay }}\left(t_{\text {ramp }}\right)\right)$, as well as the characteristic currents $\Delta I_{m}$ and $\Delta I_{n}$, change from run to run. This corresponds to the well-known fact that the snap-back (as the decay) is a function of the magnet powering history. We have found however, that the two sets of fit parameters are strongly correlated, and once represented in a scatter plot they lie on a straight line. Furthermore, a very interesting property is that the correlation between the fit parameters is approximately the same for all magnets tested. An example of this correlation on the sextupole fit parameters $b_{3}^{\text {decay }}\left(t_{\text {ramp }}\right)$ vs $\Delta I_{3}$ is shown in Fig. 9 for the 138 measurements on LHC dipoles tested to date using the snap-back analyzer [23]. This finding is substantiated by the fact that the magnets tested were not specially selected (e.g. with respect to cable properties) and comparable results are found performing the same measurements and data analysis on both the LHC and Tevatron dipoles, as discussed later. Hence, it seems that the correlation plot can be used to characterize the behavior of the dipoles in the whole accelerator, i.e., it can act as a scaling law.

The implication is that only one of the two fit parameters, either $c_{n}^{\text {decay }}\left(t_{\text {ramp }}\right)$ or $\Delta I_{n}$, is strictly necessary to predict the sextupole change.

In practice, the waveform of the snap-back can be predicted by taking the observed decay $c_{n}^{\text {decay }}\left(t_{\text {ramp }}\right)$ at the end of injection [e.g. computed using Eq. (7)], and computing the corresponding $\Delta I_{n}$ using the linear correlation coefficient $g_{n}^{\mathrm{SB}}$ :

$$
c_{n}^{\text {decay }}\left(t_{\text {ramp }}\right)=g_{n}^{\mathrm{SB}} \cdot \Delta I_{n}
$$

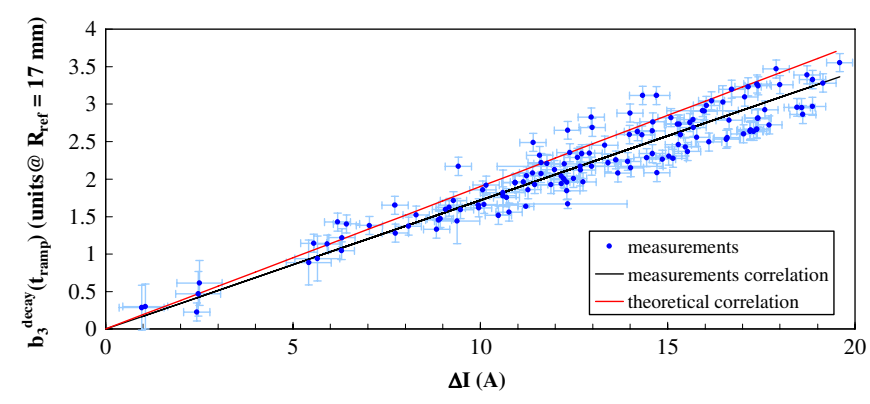

FIG. 9. (Color) Scatter plot of the sextupole fit parameters $b_{3}^{\text {decay }}\left(t_{\text {ramp }}\right)$ (units $@ R_{\text {ref }} 17 \mathrm{~mm}$ ) and $\Delta I_{n}$ that correspond to sets of different powering cycles in the LHC dipoles tested and analyzed to date. The data has been fitted with a linear regression and is compared to the theoretical prediction presented in [25]. 


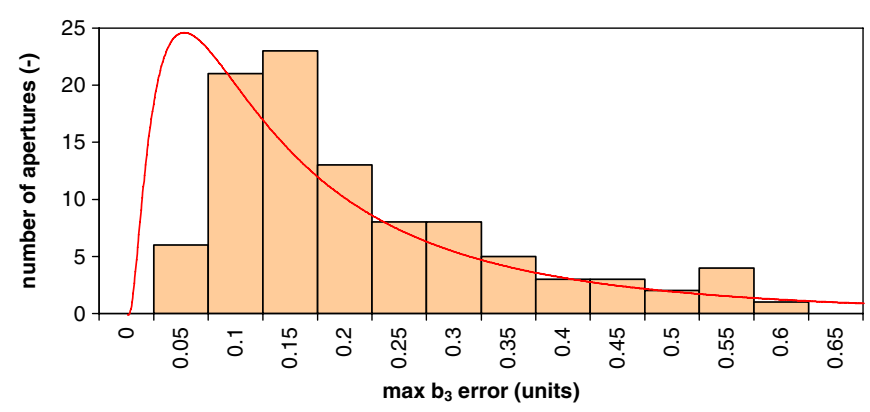

FIG. 10. (Color) The histogram and log-normal distribution of the difference between the sextupole snap-back amplitudes and the correlation line.

For the sextupole, which is in practice the only harmonic that could be sampled reliably, the value obtained from measurements is $g_{3}^{\mathrm{SB}}=0.172$ units/A which is comparable to the theoretical value $g_{3}^{\mathrm{SB}_{\text {theoretical }}}=0.19$ units $/ \mathrm{A}$ calculated in [25]. The $R$-squared value of the correlation line is 0.882 . To have a better indication of the quality of the snap-back scaling law, the same procedure as used in the decay scaling analysis described above is employed. This is done by taking the residual error as being the maximum deviation of the fit parameter $b_{3}^{\text {decay }}\left(t_{\text {ramp }}\right)$ from the correlation of Eq. (14) for all measurement sets analyzed. The histogram and the log-normal distribution of the difference between the sextupole snap-back amplitudes and the correlation line are shown in Fig. 10. The use of the log-normal distribution is justified by the same reasoning discussed earlier. The residual errors range from 0.01 to 0.6 units @ $17 \mathrm{~mm}$, with a median value of 0.14 units @ $17 \mathrm{~mm}$. The above values for the median residual error can be taken as an estimate for the deviation between the predicted and the actual snap-back waveforms in the accelerator.

\section{B. Tevatron dipoles}

In support of the above discussion, we report here a summary of the sextupole snap-back measurements of

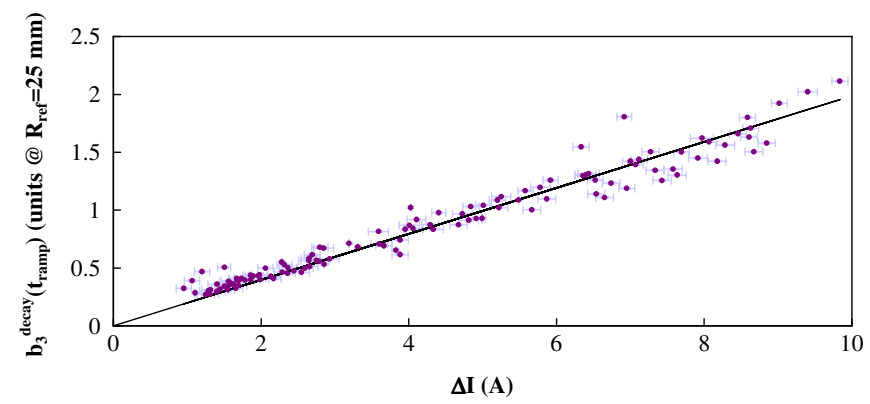

FIG. 11. (Color) Scatter plot of the fit parameters $b_{3}^{\text {decay }}\left(t_{\text {ramp }}\right)$ (units @ $R_{\text {ref }}=25 \mathrm{~mm}$ ) and $\Delta I_{3}$ that correspond to sets of different powering cycles in four Tevatron dipoles tested and analyzed to date. $g_{3}^{\mathrm{SB}}=0.198$. the same type as described above that were performed on 12 Tevatron dipoles [26]. Following the same analysis procedure as for the LHC dipoles, the result is represented in the scatter plot of Fig. 11, and leads to the same conclusion, namely, that the two parameters $c_{n}^{\text {decay }}\left(t_{\text {ramp }}\right)$ and $\Delta I_{n}$ are strongly correlated.

The fact that the same result is obtained on two different families of dipole magnets, with major design and manufacturing differences (both on the superconducting cable and coil) supports the idea that the correlation found has some fundamental origin, and can thus be used for a robust prediction.

\section{MODEL OF THE POWERING HISTORY DEPENDENCE}

The decay and snap-back of allowed multipoles in the LHC magnets is known to be strongly dependent on the powering history of the magnet $[4,5,8,9,27]$. This dependence can be explained by the way the nonuniform current distributions are formed and are diffused in the Rutherford cable during magnet powering. The studies and analysis performed over short dipole models, dipole prototypes, and series dipole magnets have concentrated on the measurement of decay and snap-back following a quench, erasing all previous memory, and a current cycle whose current values and duration have been varied parametrically. The prototype of this cycle is shown in Fig. 12, which also defines the main parameters varied.

The measurements cited above have shown that three parameters mostly affect the injection decay amplitude and subsequent snap-back. These are the flattop current $I_{\mathrm{FT}}$, the flattop time $t_{\mathrm{FT}}$, and the time spent on the preinjection plateau $t_{\text {preparation }}$.

In terms of the notation introduced in the previous section, the change in the decay amplitude can be described through a change of the parameter $\delta$ in Eq. (3), where, taking the example of the harmonic of order $n$, we have in general that

$$
\delta_{n}=\delta_{n}\left(I_{\mathrm{FT}}, t_{\mathrm{FT}}, t_{\text {preperation }}\right) .
$$

To model the changes in $\delta_{n}$, we use the following parame-

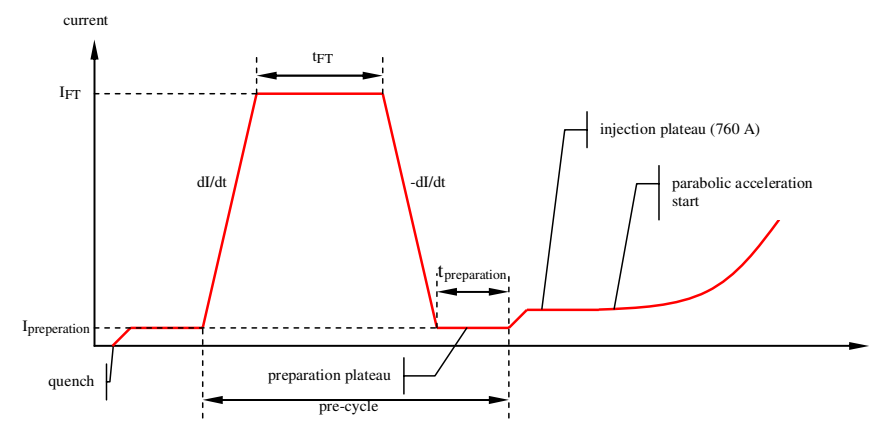

FIG. 12. (Color) A typical precycle and the main parameters defining its shape. 
trization:

$$
\begin{aligned}
\delta_{n}= & \delta_{\text {std }}\left|\frac{E_{0}^{n}-E_{1}^{n} e^{-\left[I_{\mathrm{FT}} /\left(\tau_{E}^{n} \cdot d I / d t\right)\right]}}{E_{0}^{n}-E_{1}^{n} e^{-\left[I_{\mathrm{FT}}^{s \mathrm{st}} /\left(\tau_{E}^{n} \cdot d I / d t\right)\right]}}\right| \\
& \times \frac{T_{0}^{n}-T_{1}^{n} e^{-\left(t_{\mathrm{FT}} / \tau_{T}^{n}\right)}}{T_{0}^{n}-T_{1}^{n} e^{-\left(t_{\mathrm{FT}}^{\mathrm{st} d} / \tau_{T}^{n}\right)}} \frac{P_{0}^{n}-P_{1}^{n} e^{-\left(t_{\text {preparation }} / \tau_{P}^{n}\right)}}{P_{0}^{n}-P_{1}^{n} e^{-\left(t_{\text {prepration }}^{\text {std }} / \tau_{P}^{n}\right)}},
\end{aligned}
$$

where $\delta_{\text {std }}$ is the decay measured for a standard precycle, i.e., with flattop current of $I_{\mathrm{FT}}^{\text {std }}=11850$ A, flattop time $t_{\mathrm{FT}}^{\mathrm{std}}=1000 \mathrm{~s}$, and no preinjection time $t_{\text {preparation }}^{\mathrm{std}}=0 \mathrm{~s}$. The time constants $\tau_{E}^{n}, \tau_{T}^{n}$, and $\tau_{P}^{n}$ describe the length of the magnet memory vs the flattop current, flattop time, and preinjection time, respectively. $d I / d t$ is the precycle current ramp rate which is taken to be $50 \mathrm{~A} / \mathrm{s}$ for both rampup and ramp-down. The fitting parameters in Eq. (16) are the above time constants and the variables $E_{0}^{n}, E_{1}^{n}, T_{0}^{n}, T_{1}^{n}$, $P_{0}^{n}$, and $P_{1}^{n}$. Equation (16) can be seen as a direct consequence of the assumption of exponential decay during constant current excitation, i.e., Eq. (3), where only the longest time constant has been retained for simplicity. The same equation can be applied to $\delta_{m}$.

The parametrization was tested against the measured effect of the three precycle parameters, as sampled on a total of 19 magnets, listed in Table III. When testing the influence of one parameter (e.g. the flattop current), the second and third parameters were held constant (e.g. the

TABLE III. Magnets considered in the analysis of the influ-

\begin{tabular}{|c|c|c|c|}
\hline Magnet & $I_{\mathrm{FT}}$ & $t_{\mathrm{FT}}$ & $t_{\text {preparation }}$ \\
\hline 1004 & $\sqrt{ }$ & & \\
\hline 1007 & $\sqrt{ }$ & & \\
\hline 1010 & & $\sqrt{ }$ & \\
\hline 1011 & & $\sqrt{ }$ & \\
\hline 1012 & $\sqrt{ }$ & & \\
\hline 1018 & & $\sqrt{ }$ & \\
\hline 1225 & & $\sqrt{ }$ & $\sqrt{ }$ \\
\hline 2010 & & $\sqrt{ }$ & \\
\hline 2123 & J & ل & $\sqrt{ }$ \\
\hline 2168 & & & $\sqrt{ }$ \\
\hline 2254 & $\sqrt{ }$ & & J \\
\hline 2290 & ل & & ل \\
\hline 3007 & $\sqrt{ }$ & & \\
\hline 3028 & & $\sqrt{ }$ & \\
\hline 3042 & & $\sqrt{ }$ & \\
\hline 3117 & $\sqrt{ }$ & $\sqrt{ }$ & ل \\
\hline 3130 & $\sqrt{ }$ & $\sqrt{ }$ & ل \\
\hline 3219 & $\sqrt{ }$ & ل & J \\
\hline 3284 & J & J & ل \\
\hline
\end{tabular}
ence of powering history on decay and snap-back at injection.
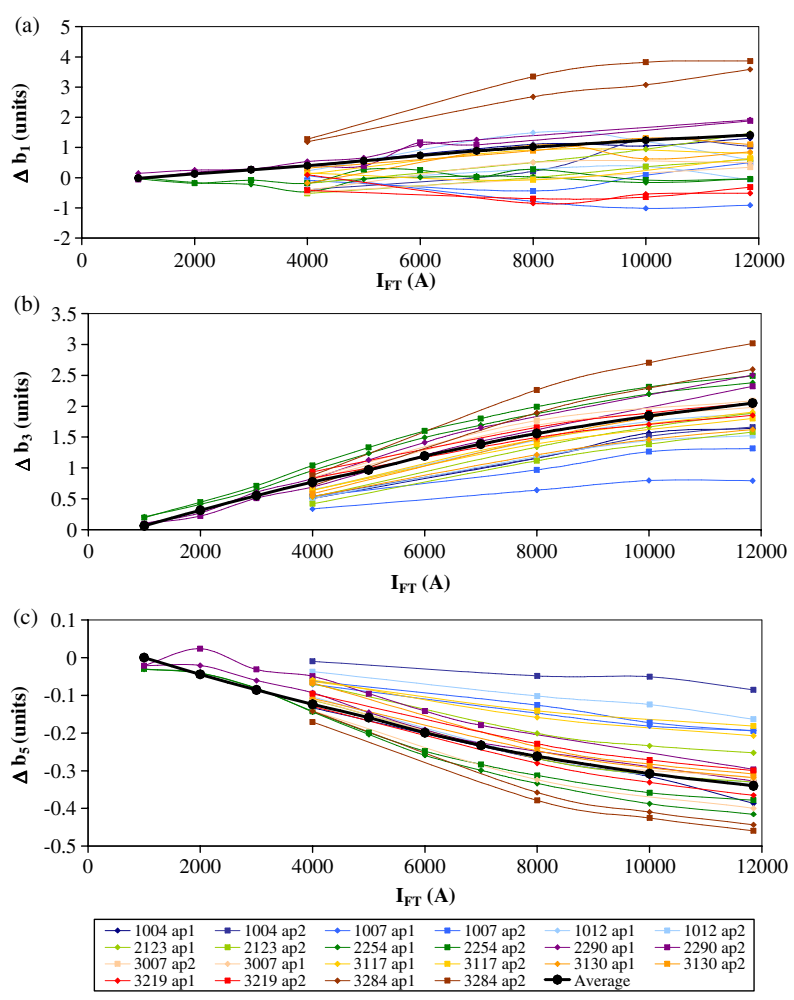

FIG. 13. (Color) The variation of the decay amplitude with flattop current for (a) $b_{1}$, (b) $b_{3}$, and (c) $b_{5}$.
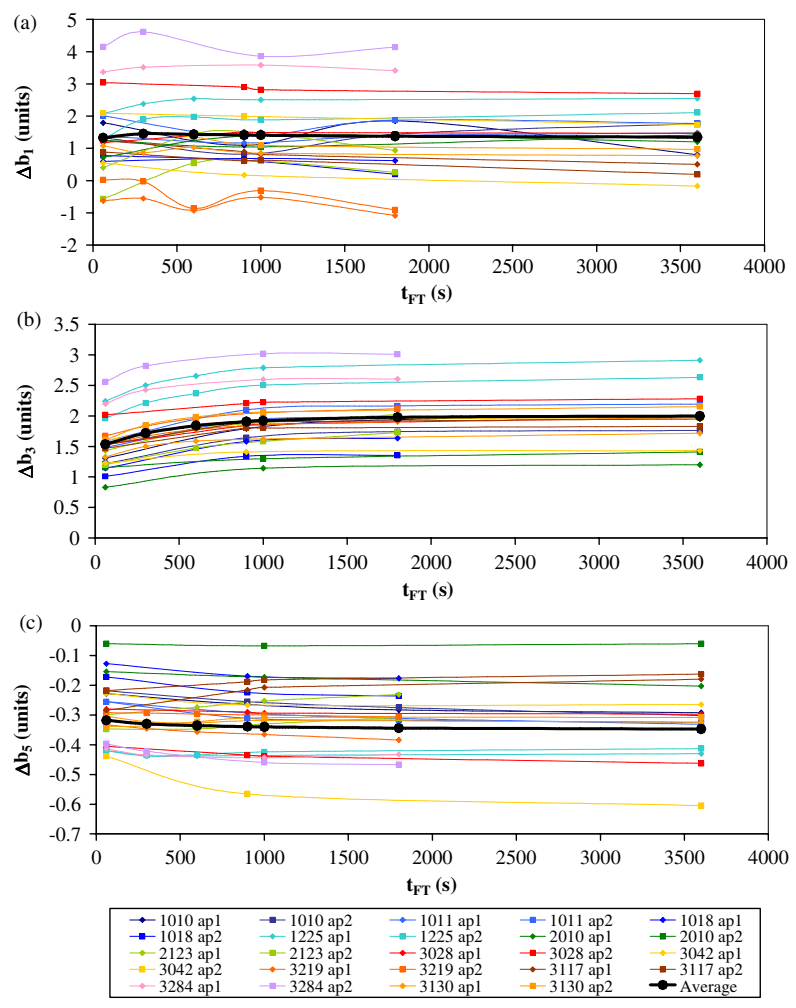

FIG. 14. (Color) The variation of the decay amplitude with flattop duration for (a) $b_{1}$, (b) $b_{3}$, and (c) $b_{5}$. 

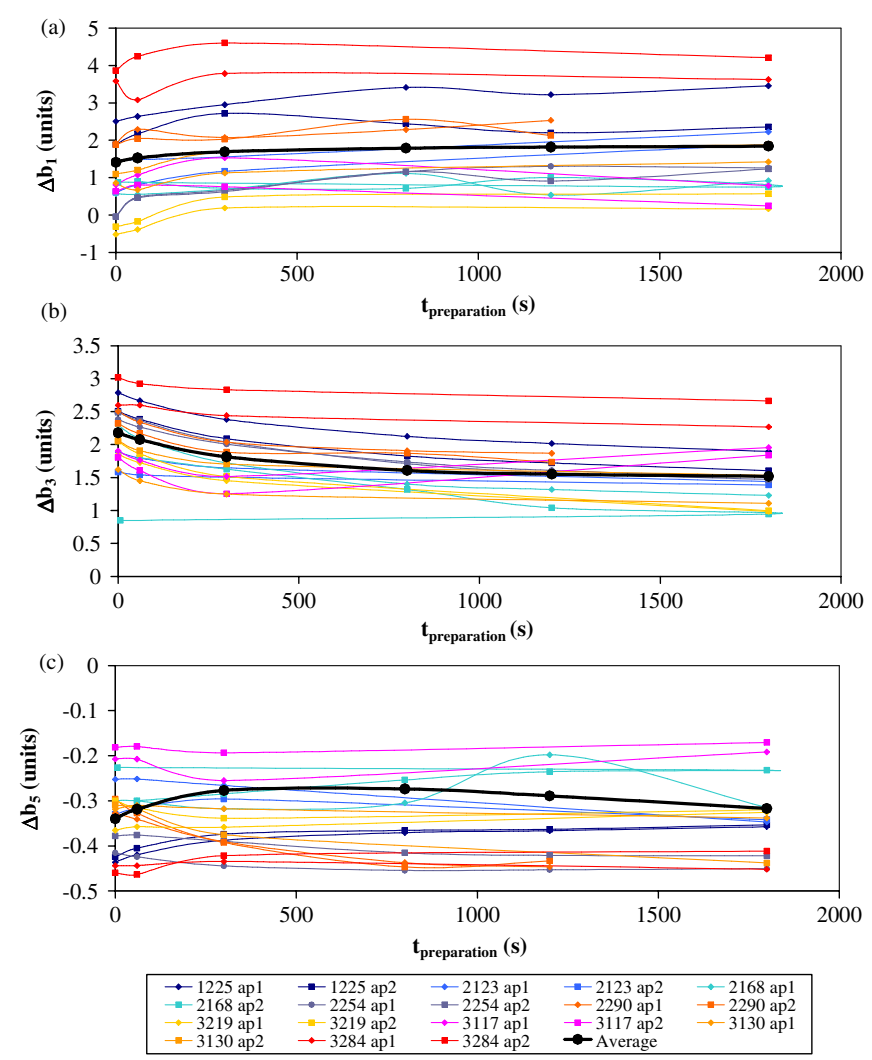

FIG. 15. (Color) The variation of the decay amplitude with preparation duration for (a) $b_{1}$, (b) $b_{3}$, and (c) $b_{5}$.

TABLE IV. The effect of each powering history parameter on the allowed harmonics.

\begin{tabular}{llll}
\hline \hline & $b_{1}$ (units) & $b_{3}$ (units) & $b_{5}$ (units) \\
\hline$I_{\mathrm{FT}}$ & 1.42 & 1.98 & 0.34 \\
$t_{\mathrm{FT}}$ & 0.02 & 0.46 & 0.03 \\
$t_{\text {preparation }}$ & 0.43 & 0.67 & 0.05 \\
\hline \hline
\end{tabular}

TABLE V. Parameters obtained fitting the model of Eq. (16) to the $b_{3}$ decay measured as a function of flattop current, flattop time, and preinjection time variations from the magnet population analyzed.

\begin{tabular}{lllll}
\hline \hline & Dimension & $b_{1}$ & $b_{3}$ & $b_{5}$ \\
\hline$E_{0}$ & $(\cdots)$ & -1.8696 & 0.0857 & -1.7896 \\
$E_{1}$ & $(\cdots)$ & -1.9930 & 0.0917 & -1.9845 \\
$\tau_{E}$ & $(\mathrm{~A})$ & 395.1 & 244.4 & 210.4 \\
$T_{0}$ & $(\cdots)$ & $\cdots$ & 1.3406 & $\cdots$ \\
$T_{1}$ & $(\cdots)$ & $\cdots$ & 0.3436 & $\cdots$ \\
$\tau_{T}$ & $(\mathrm{~s})$ & $\cdots$ & 504.9 & $\cdots$ \\
$P_{0}$ & $(\cdots)$ & $\cdots$ & 1.7472 & $\cdots$ \\
$P_{1}$ & $(\cdots)$ & $\cdots$ & -0.7568 & $\cdots$ \\
$\tau_{P}$ & $(\mathrm{~s})$ & $\cdots$ & 375.4 & $\cdots$ \\
\hline \hline
\end{tabular}

flattop time and the preinjection time) at the value corresponding to the standard precycle. In addition, it should be noted that, due to the long test time (each measurement requires a quench and a complete precycle that last several hours), in some cases only the influence of one of the three parameters was measured.

Figure 13 shows the measurement results and the average variation of decay amplitude vs precycle flattop current
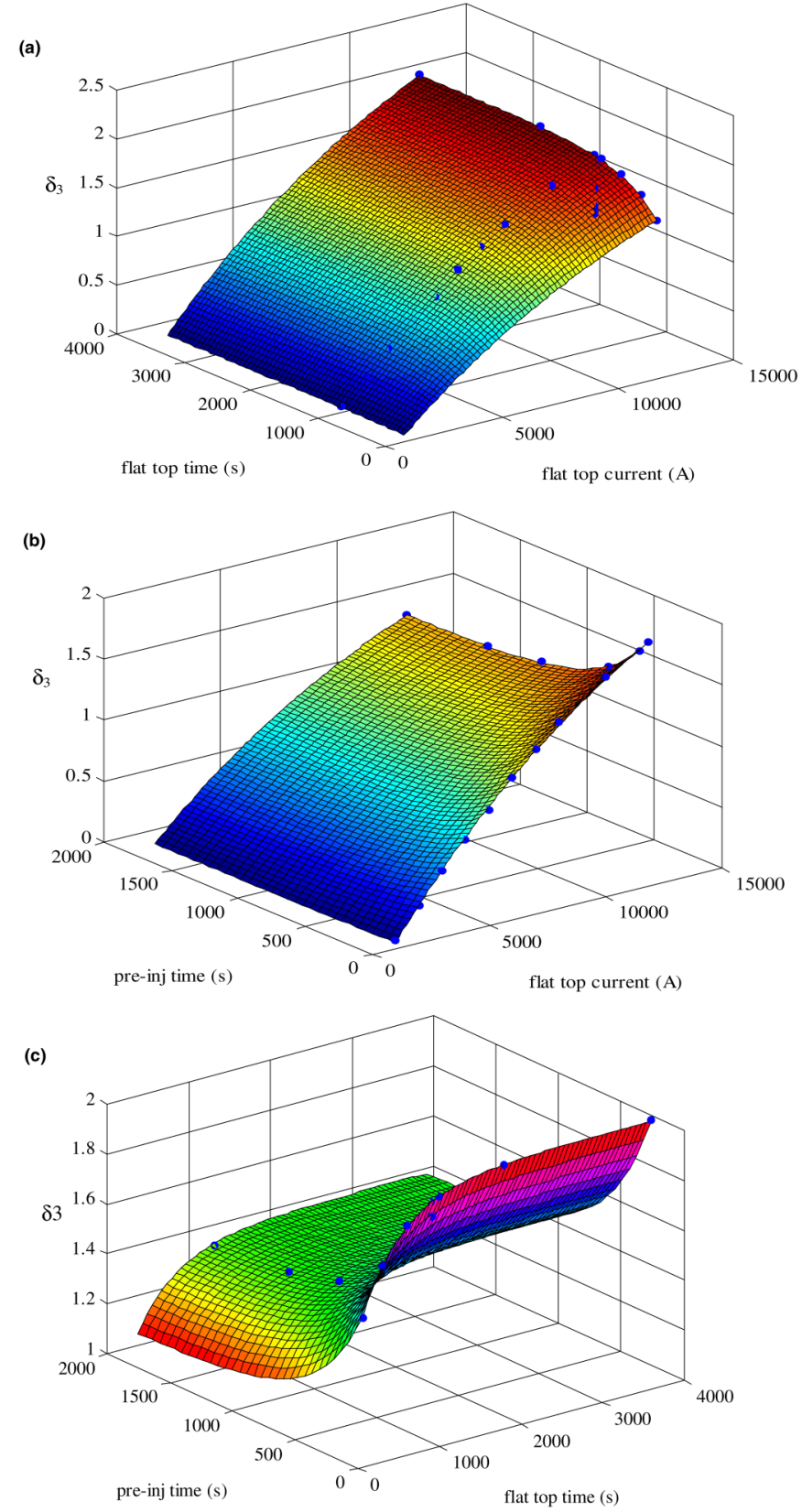

FIG. 16. (Color) Plot of the surface of $\delta_{3}$ in the space defined by variations of (a) flattop current and flattop time, (b) flattop current and preinjection time, (c) preinjection time and flattop time as generated with the parameters of Table $\mathrm{V}$ and representative for the LHC behavior. The measured values are depicted by the blue points. 
for the measurements shown in Table III. $b_{1}, b_{3}$, and $b_{5}$ all have an approximate linear dependence on $I_{\mathrm{FT}}$. We remark however that the $b_{1}$ dependence is very close to the measurement accuracy limit.

Figures 14 and 15 show the measurement results and the average variation of decay amplitude vs precycle flattop duration and the decay amplitude vs preparation duration, respectively, for the measurements shown in Table III. $b_{1}$, $b_{3}$, and $b_{5}$ all have a general asymptotic exponential dependence. However, the dependence for $b_{1}$ and $b_{5}$ in both cases is considered to be negligible since it is comparable to the rotating coils measurement repeatability and is not reproducible on a magnet by magnet basis. Therefore, these dependencies are only considered to be important for $b_{3}$.

We can assess the importance of the three precycle parameters on the main field and the harmonics being considered by comparing the range of variation of the measurements average. The effect of each powering history parameter on the decay amplitude is summarized in Table IV. The $I_{\mathrm{FT}}$ dependence is relevant for the main field, sextupole, and decapole, while in practice the other two parameters $t_{\mathrm{FT}}$ and $t_{\text {preparation }}$ only affect the sextupole. The fit of the parametrization of Eq. (16) yields the parameters
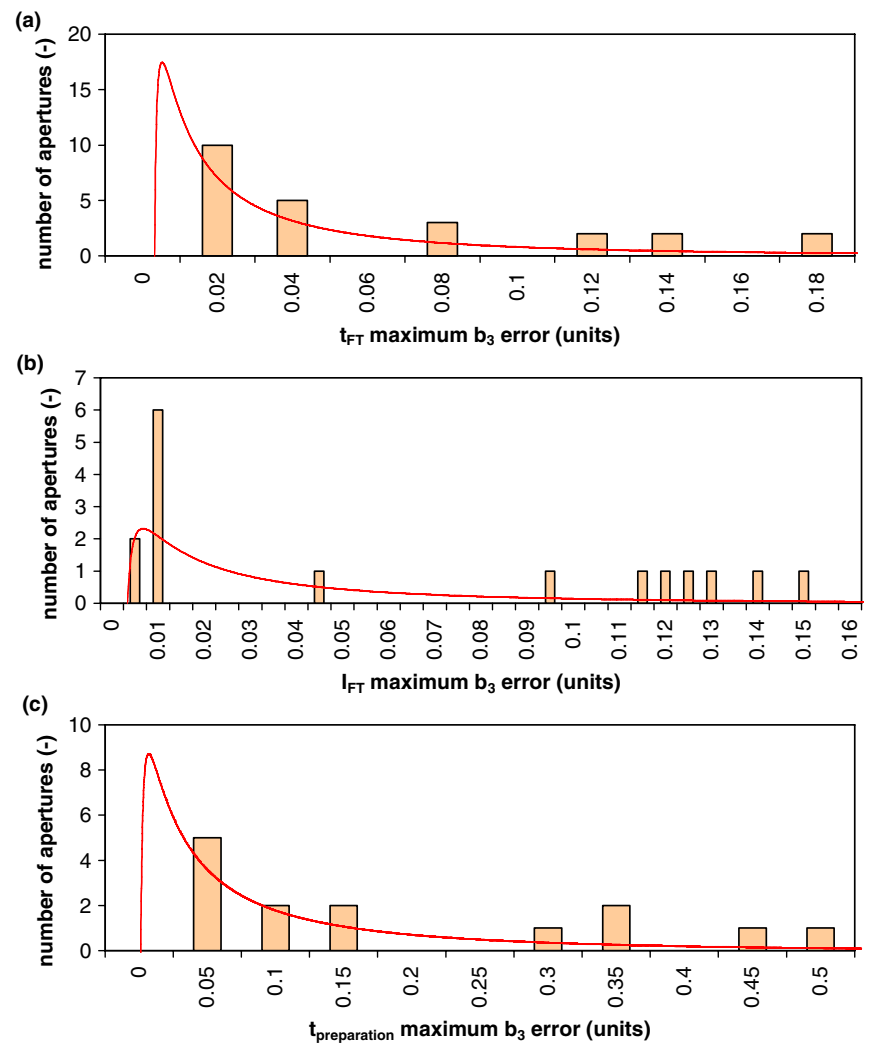

FIG. 17. (Color) (a), (b), (c) Histograms and log-normal distribution of the maximum residual error for $I_{\mathrm{FT}}, t_{\mathrm{FT}}$, and $t_{\text {preparation }}$, respectively, between the scaled harmonic decay and the average harmonic decay of the magnet set analyzed. reported in Table V. The surfaces in Fig. 16 show how the parametrization of Eq. (16) describes the average magnet data scaled to the entire magnet population using Eq. (8).

The parameters of Table V can be used in Eq. (16) to compute the difference between the scaled behavior of a single magnet [using Eq. (8)] and the 4D fits. The maximum residual error between these two can be taken as a measure of the quality of the scaling. The histograms and the log-normal distribution for the three powering history parameters are shown in Fig. 17. The use of the log-normal distributions is justified by the reasons described in Sec. II B. Because of the modest number of measurements, Fig. 17 may not indicate a log-normal distribution, however the goodness of fit is confirmed by checking with the Kolmogorov-Smirnov test [16].

As done earlier, the medians can be taken as an indication of the residual error in a magnet selected at random. For the $b_{3}$ flattop current dependence, the maximum residual error ranges from 0.004 to 0.15 units, with a median value of 0.03 units. For the $b_{3}$ flattop time dependence, the maximum residual error ranges from 0.001 to 0.18 units, with a median value of 0.02 units @ $17 \mathrm{~mm}$. For the $b_{3}$ preinjection time dependence, the maximum residual error ranges from 0.008 to 0.46 units, with a median value of 0.07 units.

For the $b_{1}$ flattop current dependence, the maximum residual scaling error ranges between 0.33 and 2.5 units with a median value of 0.835 units. For the $b_{5}$ flattop current dependence, the maximum residual scaling error ranges between 0.005 and 0.11 units with a median value of 0.016 units.

\section{CONCLUSIONS}

The decay and snap-back behavior of a set of several magnets in different magnetic states can be deduced using simple models of the data. We have given suitable mathematical models for the scaling laws, and shown how to apply them to represent a portion or the whole LHC ring. Following the discussion of our result, the basic information to establish and adapt the scaling can be derived from

TABLE VI. Summary of the maximum residual error expected due to the dynamic model and scaling procedure. (All values are in units @ $\left.R_{\text {ref }}\right)$.

\begin{tabular}{lllll}
\hline \hline & & $b_{1}$ & $b_{3}$ & $b_{5}$ \\
\hline Decay model & & 0.32 & 0.13 & 0.04 \\
Decay scaling & & 0.5 & 0.06 & 0.02 \\
Powering history scaling & $I_{\mathrm{FT}}$ & 0.835 & 0.03 & 0.016 \\
& $T_{\mathrm{FT}}$ & $\ldots$ & 0.02 & $\ldots$ \\
& $T_{\text {preparation }}$ & $\ldots$ & 0.07 & $\ldots$ \\
Total decay residual error & & 1.02 & 0.16 & 0.05 \\
Snap-back model & & $\ldots$ & 0.03 & 0.03 \\
Snap-back correlation & & $\ldots$ & 0.14 & $\ldots$ \\
Total snap-back error & & $\ldots$ & 0.14 & $\ldots$ \\
\hline \hline
\end{tabular}


(a) the series measurements in operating conditions, available on a sample of the dipole magnets, (b) extended measurements on selected magnets that will be available as an offline reference for LHC operation, and (c) direct beam measurements, e.g., taken during machine development time.

In the case of measurements on a single magnet, the residual error of the scaled predictions does not depend drastically on the magnet selected, so that the scaling of a single magnet to a portion or the whole LHC ring will not be a critical process. In practice, following the reasoning of this chapter, half of the magnets produced can be used as LHC references.

Table VI reports a summary of the maximum expected residual errors due to the dynamic model and the scaling procedure. For the injection plateau, this estimate is obtained as the quadratic sum of the residual error on the decay and on the prediction of the powering history dependence. To put these values in perspective, the maximum residual sextupole error corresponds to about 7 units of chromaticity in the LHC, which is an excellent result.

[1] N. Sammut, L. Bottura, and J. Micallef, Phys. Rev. ST Accel. Beams, 9, 012402 (2006).

[2] D. A. Finley, D. A. Edwards, R. W. Hanft, R. Johnson, A. D. Mc Inturff, and J. Strait, in Proceedings of the 12th Particle Accelerator Conference, Washington, DC, USA, 1987, pp. 151-153.

[3] L. Bottura, M. Breschi, and M. G. Fabbri, J. Appl. Phys. 92, 7571 (2002).

[4] M. Schneider, Ph.D. dissertation, Technischen Universitaet Wien, 1999.

[5] M. Haverkamp, Ph.D. dissertation, Twente University, Enchede, 2003.

[6] R. Stiening, SSCL-359, 1991.

[7] R. Wolf, in Proceedings of the 15th International Conference on Magnet Technology, Beijing, China, 1997, pp. 20-34.

[8] Ray Hanft, Bruce Brown, David Herrap, Mike Lamm, Alfred McInturff, and Mike Syphers, IEEE Trans. Magn. 25, 1647 (1989).

[9] G. Annala, P. Bauer, L. Bottura, M. A. Martens, N. Sammut, G. Velev, and V. Shiltsev, Proceedings to the Particle Accelerator Conference, Knoxville, TN, USA, 2005, pp. 54-58.

[10] H. Brück, D. Gall, G. Knies, J. Krzywinski, R. Lange, R. Meinke, P. Schmüser, and Y. Zhao, Proceedings of the European Particle Accelerator Conference, Nice, France, 1990.

[11] H. Brück, B. Holzer, C. Luettge, and B. Pawlowski, in Proceedings of the 5th European Particle Accelerator Conference, Barcelona, Spain, 1996, pp. 392-394.

[12] A. Devred, J. DiMarco, J. Kuzminski, R. Stiening, J. Tompkins, Y. Yu, H. Zheng, T. Ogitsu, R. Hanft, P. O.
Mazur, D. Orris, and T. Peterson, Proceedings to the IEEE Particle Accelerator Conference, San Francisco, California, 1991.

[13] W. Fischer, A. Jain, and S. Tepikian, Phys. Rev. ST Accel. Beams 4, 041002 (2001).

[14] L. Rossi, IEEE Trans. Appl. Supercond. 14, 153 (2004).

[15] J. Billan, L. Bottura, M. Buzio, G. D’Angelo, G. Deferene, O. Dunkel, P. Legrand, A. Rijllart, A. Siemko, P. Sievers, S. Schloss, and L. Walckiers, IEEE Trans. Appl. Supercond. 10, 1422 (2000).

[16] J.D. Gibbons, in Handbook of Statistical Methods for Engineers and Scientists, edited by H. M. Wadsworth, R.W. Hauserman, and I. M. Stochmal (McGraw-Hill, New York, 1990).

[17] G. V. Velev, G. Annala, P. Bauer, R. Carcagno, J. DiMarco, H. Glass, R. Hanft, R. Kephart, M. Lamm, M. Martens, P. Sclabach, C. Sylvester, M. Tartaglia, and J. Tompkins, Proceedings of European Accelerator Conference, Lucerne, Switzerland, 2004, pp. 1780-1782.

[18] P. Bauer, G. Ambrosio, G. E. Annala, J. DiMarco, R. W. Hanft, M. J. Lamm, M. A. Martens, P. Schlabach, D. Still, M. A. Tartaglia, J. C. Tompkins, and G. Velev, Proceedings of European Accelerator Conference, Lucerne, Switzerland, 2004, pp. 818-820.

[19] G. Annala, G. Ambrosio, P. Bauer, L. Bottura, R. Carcagno, J. DiMarco, R. W. Hanft, M. J. Lamm, M. Martens, P. Schlabach, C. Sylvester, M. Tartaglia, J. C. Tompkins, and G. Velev, Fermilab/TD Report No. TD-04043, 2004.

[20] H. Brück, D. Degele, P. D. Gall, G. Hase, R. Meinke, M. Stolper, and F. Willeke, Proceedings to the High Energy Accelerators Conference, Hamburg, 1992, Vol. 2, pp. 614-616.

[21] B. Holzer, CERN Accelerator School (CAS), CERN 9603, Hamburg, Germany, 1996, p. 123.

[22] B. Holzer and C. Montag, Proceedings to the European Accelerator Conference, Vienna, Austria, 2000, pp. 21422144.

[23] N. Sammut, E. Benedico Mora, L. Bottura, P. Galbraith, D. Giloteaux, G. Greco, M. Haverkamp, M. Marchesotti, A. Masi, J. Micallef, T. Pieloni, N. Smirnov, and A. Tikhov, Proceedings of the Instrumentation and Measurement Technology Conference, Sorrento, Italy, 2006.

[24] L. Bottura, T. Pieloni, S. Sanfilippo, G. Ambrosio, P. Bauer, and M. Haverkamp, Proceedings of European Accelerator Conference, Lucerne, Switzerland, 2004, pp. 1609-1611.

[25] L. Bottura, G. Ambrosio, P. Bauer, M. Haverkamp, T. Pieloni, S. Sanfilippo, and G. Velev, IEEE Trans. Appl. Supercond. 15, 1217 (2005).

[26] G. Velev, P. Bauer, R. Carcagno, M. Lamm, D. Orris, P. Sclabach, C. Sylvester, M. Tartaglia, and J. Tompkins, in Proceedings of the European Particle Conference, Edinburgh, UK, 2006, pp. 2640-2642.

[27] L. Bottura, L. Walckiers, and R. Wolf, IEEE Trans. Appl. Supercond. 7, 602 (1997). 